Article

\title{
Comparison of Prognostic Impact between the Child-Pugh Score and Skeletal Muscle Mass for Patients with Liver Cirrhosis
}

\author{
Hiroki Nishikawa, Hirayuki Enomoto *, Akio Ishii, Yoshinori Iwata, Yuho Miyamoto, \\ Noriko Ishii, Yukihisa Yuri, Kunihiro Hasegawa, Chikage Nakano, Takashi Nishimura, \\ Kazunori Yoh, Nobuhiro Aizawa, Yoshiyuki Sakai, Naoto Ikeda, Tomoyuki Takashima, \\ Ryo Takata, Hiroko Iijima and Shuhei Nishiguchi \\ Department of Hepatobiliary and Pancreatic disease, Department of Internal Medicine, \\ Hyogo College of Medicine, 1-1, Mukogawacho, Nishinomiyashi, Hyogo 663-8501, Japan; \\ nishikawa_6392@yahoo.co.jp (H.N.); akio0010@yahoo.co.jp (A.I.); yo-iwata@hyo-med.ac.jp (Y.I.); \\ yuho.0818.1989@gmail.com (Y.M.); ishinori1985@yahoo.co.jp (N.I.); gyma27ijo04td@gmail.com (Y.Y.); \\ hiro.red1230@gmail.com (K.H.); chikage@hyo-med.ac.jp (C.N.); tk-nishimura@hyo-med.ac.jp (T.N.); \\ mm2wintwin@ybb.ne.jp (K.Y.); nobu23hiro@yahoo.co.jp (N.A.); sakai429@hyo-med.ac.jp (Y.S.); \\ nikeneko@hyo-med.ac.jp (N.I.); tomo0204@yahoo.co.jp (T.T.); chano_chano_rt@yahoo.co.jp (R.T.); \\ hiroko-i@hyo-med.ac.jp (H.I.); nishiguc@hyo-med.ac.jp (S.N.) \\ * Correspondence: enomoto@hyo-med.ac.jp; Tel.: +81-798-45-6111; Fax: +81-798-45-6608
}

Received: 13 May 2017; Accepted: 9 June 2017; Published: 12 June 2017

\begin{abstract}
Aims: To investigate the influence of skeletal muscle mass index (SMI) as determined by bioimpedance analysis (BIA) (appendicular skeletal muscle mass / (height) ${ }^{2}$ ) on survival by comparing the Child-Pugh score in patients with liver cirrhosis (LC, $n=383$, average age $=65.2$ years). Patients and methods: In terms of comparison of the effects of SMI and other markers on survival, we used time-dependent receiver operating characteristics (ROC) analysis. Results: The average SMI for male was $7.4 \mathrm{~cm}^{2} / \mathrm{m}^{2}$ whereas that for female was $6.0 \mathrm{~cm}^{2} / \mathrm{m}^{2}(p<0.0001)$. As for the Child-Pugh score, five points were in the majority, both in males $(51.7 \%,(106 / 205))$ and females $(44.9 \%,(80 / 178))$. For both genders, the survival curve was well stratified according to SMI $(p<0.0001$ for males and $p=0.0056$ for females). In the multivariate analysis for survival, SMI and Child-Pugh scores were found to be significant both in males and females. In time-dependent ROC analyses, all area under the ROCs (AUROCs) for SMI in each time point were higher than those for Child-Pugh scores in males, while in females AUROCs for Child-Pugh scores at each time point were higher than those for SMI. Conclusion: SMI using BIA can be helpful for predicting outcomes, at least in male LC patients.
\end{abstract}

Keywords: liver cirrhosis; bioimpedance analysis; skeletal muscle mass index; Child-Pugh score; prognosis

\section{Introduction}

The liver is the pivotal organ for metabolism and it metabolizes carbohydrates, lipids, and proteins, which are the so-called "three major nutrients" [1-4]. Liver cirrhosis (LC) is an end-stage form in liver diseases and LC is characterized by several metabolic or nutritional disorders and portal hypertension-related complications such as ascites or varices, all of which can lead to dismal clinical outcome [1-4]. Over the past two or three decades, numerous clinical and biochemical predictors have been proposed in an effort to more accurately predict the prognosis in LC patients and evaluate their short and long-term survival correctly [5-9]. The Child-Pugh scoring system and the Model for End-stage Liver Disease (MELD) scoring system are two major prognostic scoring systems in LC patients [5-9]. In particular, the MELD score is calculated by three easily available and reproducible 
laboratory tests and is useful for predicting outcomes for patients undergoing liver transplantation (LT) [5]. In our country, LT is not common due to the shortage of transplanted liver and the Child-Pugh scoring system has been preferably used for assessing prognosis in LC patients.

Sarcopenia is a clinical entity as determined by skeletal muscle mass loss and decline of muscle strength and this clinical entity has recently drawn much attention among clinicians owing to its significant deleterious impact on outcomes [10-16]. LC can be associated with secondary sarcopenia because of protein metabolic disorder and/or energy metabolic disorder [11,14,15]. Skeletal muscle mass loss can be linked to poorer clinical outcomes in LC patients, hepatocellular carcinoma (HCC) patients, or patients with other malignancies [17-26]. Skeletal muscle mass can be assessed by computed tomography (CT), magnetic resonance imaging (MRI), dual energy X-ray absorptiometry and bioimpedance analysis (BIA), which are consistent and accurate assessment modalities $[14,15,17,19,27,28]$. Among these modalities, BIA is particularly attractive since it can noninvasively determine body composition analysis in LC patients $[14,15,17,19,27,28]$.

However, which of two prognostic markers (i.e., the Child-Pugh scoring system and skeletal muscle mass) has stronger influence on clinical outcomes in patients with LC remains unclear. Addressing these questions may be clinically of significance. The aim of this study was to investigate the influence of skeletal muscle mass as determined by data in BIA on survival compared with the Child-Pugh score in patients with LC.

\section{Patients and Methods}

\subsection{Patients}

The current study was a single center retrospective study. Between October 2005 and October 2015, a total of 529 LC individuals with BIA data available were admitted at the Division of Hepatobiliary and Pancreatic disease, Department of Internal Medicine, Hyogo College of Medicine, Hyogo, Japan. In our department, BIA (Inbody 720, Tokyo, Japan) was routinely performed in the resting and standing position principally on an outpatient basis for patients who consented to nutritional evaluation. In this analysis, skeletal muscle mass was evaluated using BIA data. Of these patients, patients with severe ascites $(n=24)$ were excluded from this study as body weight, body mass index (BMI), and skeletal muscle mass index (SMI) using BIA may be overestimated in these patients $[29,30]$. Twenty-three subjects had been lost to follow-up within one year after performing BIA and they were excluded from this analysis for avoiding bias. In the remaining 482 subjects, 99 had HCC on radiological findings at baseline and they were also excluded because presence of HCC can affect the interpretation of BIA data. A total of 383 subjects were therefore analyzed in the current study. Follow-up observation after BIA included periodical blood tests, radiological assessments by ultrasonography (US), CT, or MRI in order to detect HCC incidence every 3-6 months. There was no patient who underwent LT during observation period. LC was diagnosed using pathological findings, radiological findings such as US, CT, or MRI and/or laboratory data including liver fibrosis markers [31-33]. In patients with lower serum albumin level (less than $3.5 \mathrm{~g} / \mathrm{dL}$ ), liver supporting therapies including branched-chain amino acid (BCAA) treatment or late evening snack with BCAA enriched snacks were in consideration $[4,34,35]$. In patients with hepatitis virus-related LC, antiviral treatments including direct acting antivirals, interferon-based regimens or nucleoside analogues therapy were also in consideration [4,34]. SMI was calculated as reported elsewhere [28]. Briefly, SMI was defined as "appendicular skeletal muscle mass/(height $(\mathrm{m}))^{2}$ " [28]. We retrospectively investigated the influence of SMI on survival in males and females, as compared with Child-Pugh scores, which was well established prognostic marker [7-9]. In terms of the comparison of the effects of SMI and other markers on survival, we used time-dependent receiver operating characteristics (ROC) analysis [36]. We also investigated parameters associated with overall survival (OS) in the univariate and multivariate analyses. HCC diagnosis and treatment choices for HCC were as reported elsewhere [37,38]. 
The ethical committee meeting in Hyogo College of Medicine acknowledged the current study protocol and this study strictly followed all regulations of the Declaration of Helsinki.

\subsection{Statistical Analyses}

Categorical parameters were compared by Fisher's exact test. Continuous parameters were compared by unpaired $t$-test, Mann-Whitney $U$ test, or Kruskal-Wallis test, as applicable. In continuous parameters, ROC curve analysis for survival was conducted for the purpose of setting the optimal cutoff point that is linked to maximal sum of specificity and sensitivity and we classified continuous parameters into two groups using these cutoff points, which was then treated as dichotomous covariates in the univariate analysis. Survival curve was created by using the Kaplan-Meier method and compared in the log-rank test. Parameters with $p$ value $<0.05$ in the univariate analysis were finally subjected to the multivariate analysis in the Cox proportional hazards model. OS was defined as the time interval from the date of performing BIA until death from any cause or the last follow-up visit. Additionally, we analyzed time-dependent ROC curves of SMI, Child-Pugh scores, and variables which revealed to be significant in the multivariate analysis for survival, and compared between area under the ROCs (AUROCs) for above parameters in each time point (two-, three-, four-, five-, six-, and seven-years) [36].

Data are shown as the average \pm standard deviation (SD) unless otherwise mentioned. Statistical significance was set at $p<0.05$. Statistical analysis was performed with the JMP 11 (SAS Institute Inc., Cary, NC, USA).

\section{Results}

\subsection{Baseline Characteristics}

The baseline characteristics of the analyzed subjects $(n=383)$ are presented in Table 1 . They included 205 males and 178 females with an average \pm SD age of $65.2 \pm 10.3$ years. The median follow-up periods were 3.2 years (range: $0.2-10.7$ years). The average \pm SD value in SMI for male was $7.4 \pm 0.9 \mathrm{~cm}^{2} / \mathrm{m}^{2}$ whereas that for female was $6.0 \pm 0.7 \mathrm{~cm}^{2} / \mathrm{m}^{2}(p<0.0001)$. According to the Asian Working Group for Sarcopenia criteria (AWGS), the cut-off values for SMI are $7.0 \mathrm{~kg} / \mathrm{m}^{2}$ for male and $5.7 \mathrm{~kg} / \mathrm{m}^{2}$ for female. [28] The proportion of decreased SMI (D-SMI: less than each cutoff value as defined by AWGS criteria) in male was $36.1 \%(74 / 205)$ and that in female was $34.8 \%(62 / 178)$. A total of 136 patients (35.5\%) had D-SMI. As for Child-Pugh scores, five points was in the majority, both in males $(51.7 \%,(106 / 205))$ and females $(44.9 \%,(80 / 178))$. In males, SMI significantly correlated with age (overall significance, $p<0.0001$ ), while in females it did not (overall significance, $p=0.1921$ ) (Figure 1A,B). In both males and females, SMI significantly correlated with BMI ( $p$ values, both $<0.0001$ ) (Figure 1C,D). In both males $(p=0.3716)$ and females $(p=0.1330)$, SMI did not significantly correlate with the Child-Pugh classification (Figure 1E,F).

\subsection{Cumulative OS Rates for the Entire Cohort, Male and Female According to SMI}

For the entire cohort $(n=383)$, the one-, three-, and five-year cumulative OS rates were $92.7 \%$, $82.4 \%$, and $59.2 \%$, respectively, in patients with D-SMI, and $97.2 \%, 92.2 \%$, and $84.4 \%$, respectively, in patients without D-SMI $(p<0.0001)$ (Figure 2). For males $(n=205)$, the one-, three-, and five-year cumulative OS rates were $91.9 \%, 78.0 \%$, and $53.6 \%$, respectively, in patients with D-SMI, and $96.2 \%$, $92.0 \%$, and $84.7 \%$, respectively, in patients without D-SMI $(p<0.0001)$ (Figure $3 \mathrm{~A})$. For females $(n=178)$, the one-, three-, and five-year cumulative OS rates were $93.6 \%, 87.7 \%$, and $66.1 \%$, respectively, in patients with D-SMI, and $98.3 \%, 92.4 \%$, and $83.8 \%$, respectively, in patients without D-SMI $(p=0.0056)$ (Figure 3B). 
Table 1. Baseline characteristics $(n=383)$.

\begin{tabular}{ccccc}
\hline Variables & $\begin{array}{c}\text { Number or } \\
\text { Average } \pm \text { SD }\end{array}$ & Male $(\boldsymbol{n}=\mathbf{2 0 5})$ & Female $(\boldsymbol{n}=\mathbf{1 7 8})$ & $\begin{array}{c}p \text { Value (Male } \\
\text { vs. Female) }\end{array}$ \\
\hline Age (years) & $65.2 \pm 10.3$ & $64.1 \pm 10.8$ & $66.4 \pm 9.5$ & 0.0403 \\
Body mass index $\left(\mathrm{kg} / \mathrm{m}^{2}\right)$ & $23.3 \pm 3.9$ & $23.4 \pm 3.8$ & $23.2 \pm 4.0$ & 0.6028 \\
Skeletal muscle mass index $\left(\mathrm{cm}^{2} / \mathrm{m}^{2}\right)$ & $6.7 \pm 1.1$ & $7.4 \pm 0.9$ & $6.0 \pm 0.7$ & $<0.0001$ \\
Causes of liver disease & $32 / 235 / 116$ & $22 / 122 / 61$ & $10 / 113 / 55$ & 0.2043 \\
Hepatitis B/Hepatitis C/others & $186 / 93 / 58 / 28 / 13 / 3 / 2$ & $106 / 42 / 31 / 15 / 6 / 3 / 2$ & $80 / 51 / 27 / 13 / 7 / 0 / 0$ & 0.2587 \\
Child-Pugh scores, 5/6/7/8/9/10/11 & $1.3 \pm 1.1$ & $1.3 \pm 1.3$ & $1.2 \pm 0.7$ & 0.2319 \\
Total bilirubin $(\mathrm{mg} / \mathrm{dL})$ & $3.7 \pm 0.53$ & $3.7 \pm 0.54$ & $3.6 \pm 0.51$ & 0.3897 \\
Serum albumin $(\mathrm{g} / \mathrm{dL})$ & $77.0 \pm 13.8$ & $77.5 \pm 13.4$ & $76.4 \pm 14.2$ & 0.4532 \\
Prothrombin time $(\%)$ & $10.6 \pm 5.5$ & $10.5 \pm 5.3$ & $10.7 \pm 5.7$ & 0.8093 \\
Platelets $\left.\left(\times 10^{4} / \mathrm{mm}\right)^{3}\right)$ & $139.7 \pm 2.5$ & $139.3 \pm 2.5$ & $140.2 \pm 2.6$ & 0.0004 \\
Serum sodium $(\mathrm{mmol} / \mathrm{L})$ & $0.74 \pm 0.51$ & $0.84 \pm 0.65$ & $0.62 \pm 0.22$ & $<0.0001$ \\
Serum creatinine $(\mathrm{mg} / \mathrm{dL})$ & $154.0 \pm 36.8$ & $152.8 \pm 35.4$ & $155.2 \pm 38.4$ & 0.5250 \\
Total cholesterol $(\mathrm{mg} / \mathrm{dL})$ & $90.5 \pm 45.1$ & $95.5 \pm 53.0$ & $84.8 \pm 33.0$ & 0.2144 \\
Triglyceride $(\mathrm{mg} / \mathrm{dL})$ & $49.3 \pm 34.8$ & $49.4 \pm 32.3$ & $49.2 \pm 37.5$ & 0.9417 \\
AST (IU/L) & $42.6 \pm 38.9$ & $43.9 \pm 34.2$ & $41.0 \pm 43.7$ & 0.0951 \\
ALT (IU/L) & $110.9 \pm 34.7$ & $114.8 \pm 39.9$ & $106.4 \pm 27.0$ & 0.0101 \\
Fasting blood glucose (mg/dL) & $44 / 339$ & $25 / 180$ & $19 / 159$ & 0.7484 \\
Ascites, yes/no & &
\end{tabular}

Data are expressed as number or average \pm standard deviation (SD). AST: aspartate aminotransferase; ALT: alanine aminotransferase.

A

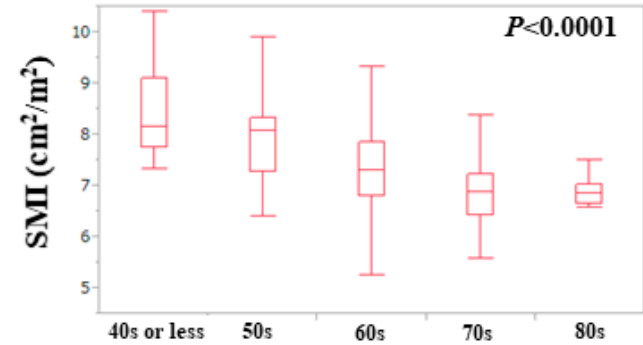

C

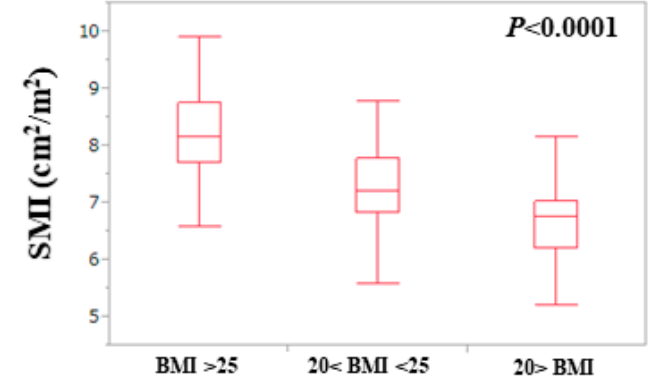

$\mathbf{E}$

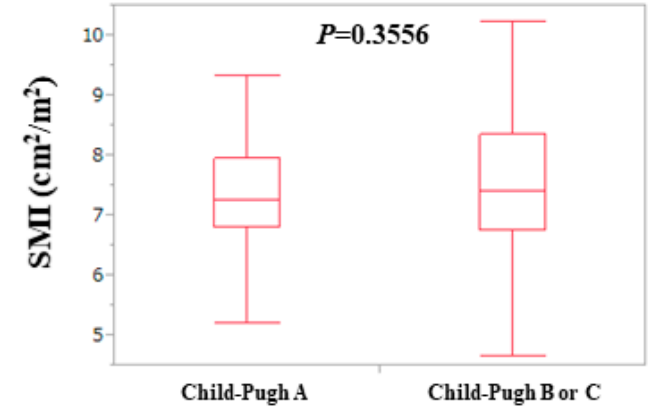

B

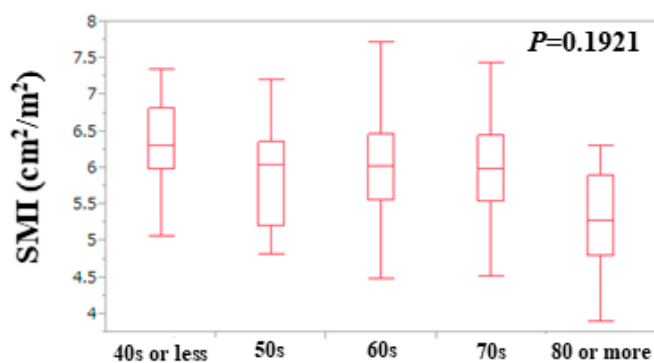

D

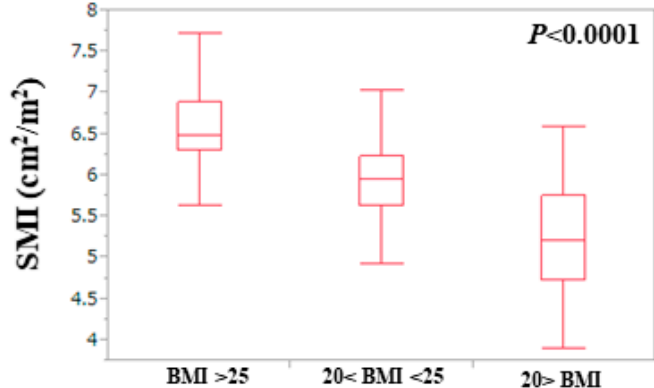

$\mathbf{F}$

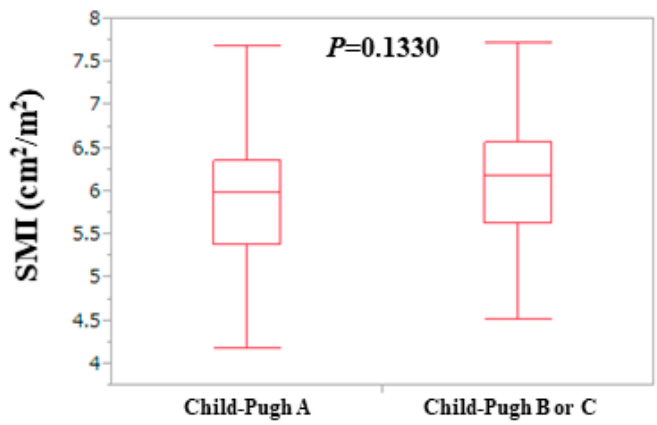

Figure 1. (A,B) Relationship between SMI and age in males (A) and females (B). (C,D) Relationship between SMI and BMI in males (C) and females (D). (E,F) Relationship between SMI and Child-Pugh classification in males (E) and females (F). SMI: skeletal muscle mass index; BMI: body mass index. 


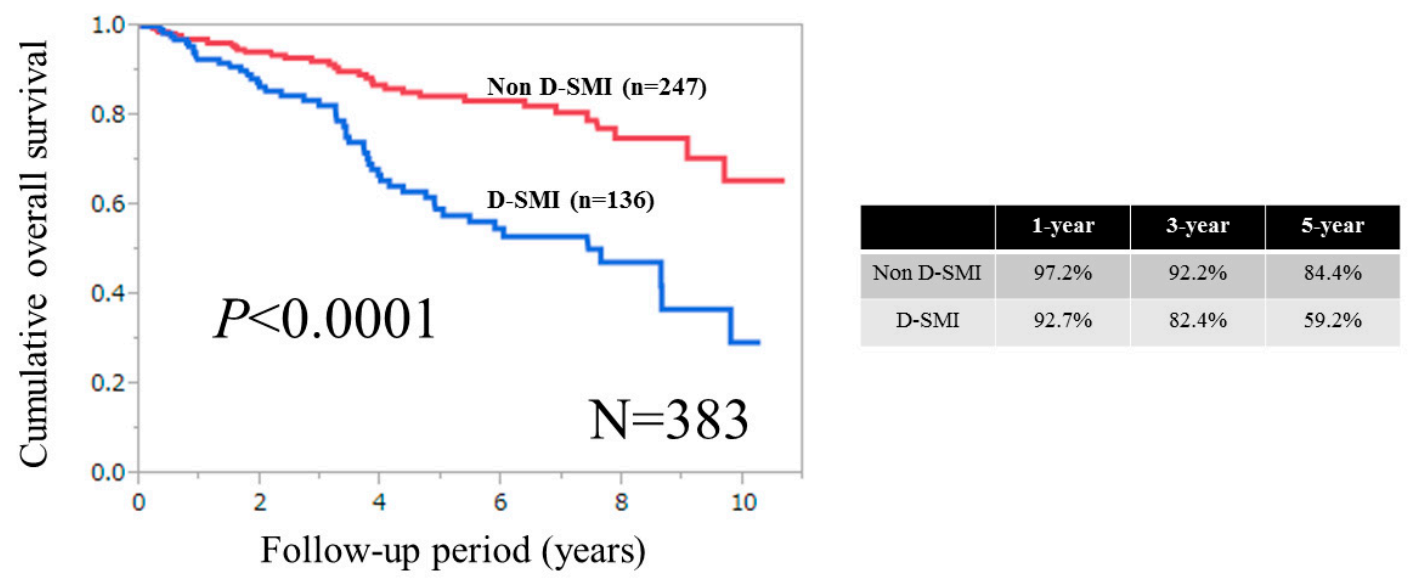

Figure 2. Cumulative overall survival for the entire cohort $(n=383)$. The one-, three-, and five-year cumulative OS rates were $92.7 \%, 82.4 \%$ and $59.2 \%$, respectively, in patients with decreased SMI (D-SMI), and $97.2 \%, 92.2 \%$ and $84.4 \%$, respectively, in patients without D-SMI $(p<0.0001)$. D-SMI was defined according to the Asian Working Group for Sarcopenia criteria. The cut-off values for SMI are $7.0 \mathrm{~kg} / \mathrm{m}^{2}$ for males and $5.7 \mathrm{~kg} / \mathrm{m}^{2}$ for females. SMI: skeletal muscle mass index; D-SMI: decreased SMI.

(A)

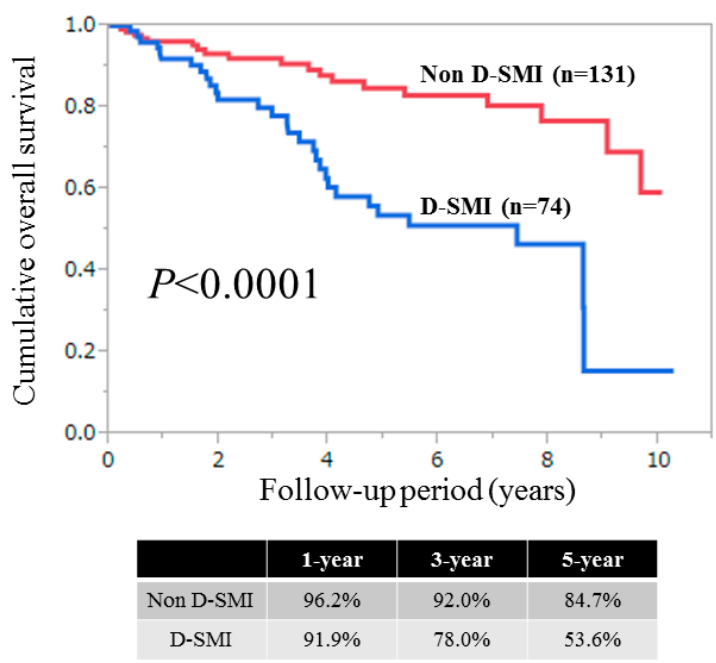

(B)

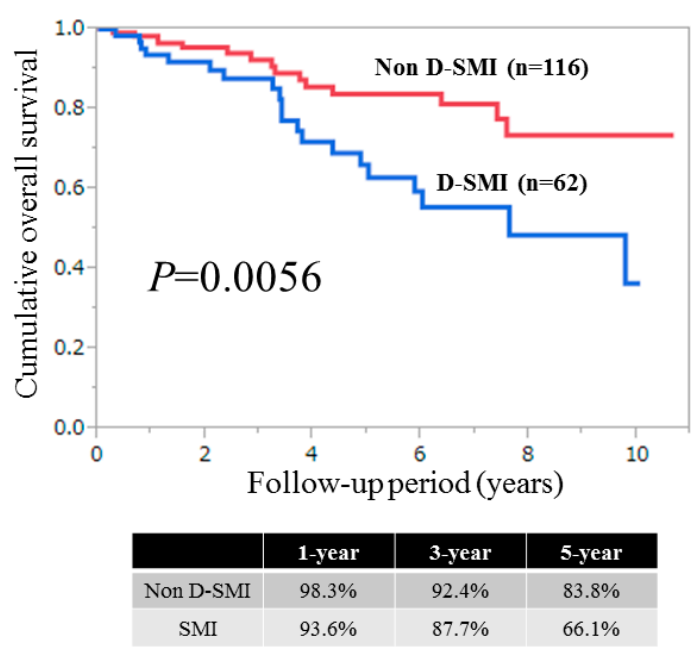

Figure 3. Cumulative overall survival for males (A) $n=205$ and for females (B) $n=178$ in patients with and without D-SMI. SMI: skeletal muscle mass index; D-SMI: decreased SMI.

Using ROC analysis for survival, the optimal cut-off point for SMI was $7.0 \mathrm{~cm}^{2} / \mathrm{m}^{2}$ in males $($ AUROC $=0.672$, sensitivity $=64.6 \%$, specificity $=69.4 \%)$ and $5.4 \mathrm{~cm}^{2} / \mathrm{m}^{2}$ in females $($ AUROC $=0.658$, sensitivity $=45.7 \%$, specificity $=83.9 \%$ ). When the cut-off value of $5.4 \mathrm{~cm}^{2} / \mathrm{m}^{2}$ was adapted to our female patients (cut-off value for female in WAGS; $5.7 \mathrm{~cm}^{2} / \mathrm{m}^{2}$ ), similar results were obtained. That is, patients with D-SMI had significantly poorer survival rates than those without D-SMI $(p=0.0076)$.

\subsection{Causes for Death for Males and Females}

In male, during the observation period, 48 patients $(23.4 \%)$ died. The causes for death were liver failure in 28 patients, HCC progression in 10 patients and miscellaneous causes in 10 patients. In female, during the observation period, 35 patients (19.7\%) died. The causes for death were liver failure in 28 patients, HCC progression in four patients and miscellaneous causes in three patients. 


\subsection{Univariate and Multivariate Analyses of Parameters Contributing to OS for Males}

Univariate analysis identified the following factors as significantly associated with OS for males: age $(p=0.0041)$; SMI $(p<0.0001)$; Child-Pugh score $(p=0.0252)$; aspartate aminotransferase $(p=0.0150)$; alanine aminotransferase (ALT) $(p=0.0407)$; serum albumin $(p=0.0011)$; serum sodium $(p=0.0133)$; serum creatinine $(p=0.0088)$; and BMI $(p=0.0041)$ (Table 2a). Since the Child-Pugh score includes serum albumin, it was not entered into the multivariate analysis, and since age and BMI significantly correlated with SMI, they were also excluded in the multivariate analysis to avoid the effect of collinearity. The hazard ratios (HRs) and 95\% confidence intervals (CIs) calculated by using multivariate analysis for the six significant variables $(p<0.05)$ in the univariate analysis are presented in Table 2b. SMI $(p=0.0005)$ and Child-Pugh score $(p=0.0424)$ were found to be significant predictors related to OS in the multivariate analysis (Table $2 b$ ).

Table 2. (a) Univariate analyses of factors linked to overall survival for males $(n=205)$. (b) Multivariate analyses of factors linked to overall survival for male.

\begin{tabular}{|c|c|c|}
\hline \multirow{3}{*}{ Variables } & \multicolumn{2}{|c|}{ a) } \\
\hline & \multirow{2}{*}{ Number of Each Category } & \multirow{2}{*}{$\begin{array}{c}\text { Univariate } \\
p \text { Value }\end{array}$} \\
\hline & & \\
\hline Age (years) $\geq 70$, yes $/$ no & $91 / 114$ & 0.0041 \\
\hline Cause of liver diseases, $\mathrm{B} / \mathrm{C} /$ others & $22 / 122 / 61$ & 0.2746 \\
\hline $\mathrm{SMI} \geq 7.0 \mathrm{~cm}^{2} / \mathrm{m}^{2}$, yes $/$ no & $127 / 78$ & $<0.0001$ \\
\hline Child-Pugh score $\geq 6$, yes $/$ no & $99 / 106$ & 0.0252 \\
\hline $\mathrm{AST} \geq 29 \mathrm{IU} / \mathrm{L}$, yes $/$ no & $146 / 59$ & 0.0150 \\
\hline $\mathrm{ALT} \geq 47 \mathrm{IU} / \mathrm{L}$, yes $/$ no & $63 / 142$ & 0.0407 \\
\hline Serum albumin $\geq 3.7 \mathrm{~g} / \mathrm{dL}$, yes $/$ no & $113 / 92$ & 0.0011 \\
\hline Total bilirubin $\geq 2.0 \mathrm{mg} / \mathrm{dL}$, yes $/ \mathrm{no}$ & $29 / 176$ & 0.1539 \\
\hline Prothrombin time $\geq 77.1 \%$, yes $/$ no & $111 / 94$ & 0.4521 \\
\hline Platelet count $\geq 9.4 \times 10^{4} / \mathrm{mm}^{3}$, yes $/$ no & $101 / 104$ & 0.2360 \\
\hline Total cholesterol $\geq 124 \mathrm{mg} / \mathrm{dL}$, yes $/$ no & $165 / 40$ & 0.2398 \\
\hline Triglyceride $\geq 56 \mathrm{mg} / \mathrm{dL}$, yes $/$ no & $170 / 35$ & 0.0649 \\
\hline Serum sodium $\geq 138 \mathrm{mmol} / \mathrm{L}$, yes $/ \mathrm{no}$ & $166 / 39$ & 0.0133 \\
\hline Fasting blood glucose $\geq 97 \mathrm{mg} / \mathrm{dL}$, yes $/$ no & $145 / 60$ & 0.4942 \\
\hline Serum creatinine $\geq 0.78 \mathrm{mg} / \mathrm{dL}$, yes $/$ no & $84 / 121$ & 0.0088 \\
\hline Body mass index $\geq 23.4 \mathrm{~kg} / \mathrm{m}^{2}$, yes $/$ no & $91 / 114$ & 0.0041 \\
\hline Ascites, yes/no & $25 / 180$ & 0.0712 \\
\hline
\end{tabular}

(b)

\begin{tabular}{|c|c|c|c|}
\hline \multirow{2}{*}{ Variables } & \multicolumn{3}{|c|}{ Multivariate Analysis } \\
\hline & Hazard Ratio & $95 \% \mathrm{CI}$ & $p$ Value \\
\hline SMI (per one $\mathrm{cm}^{2} / \mathrm{m}^{2}$ ) & 0.571 & $0.416-0.777$ & 0.0005 \\
\hline AST (per one IU /L) & 1.005 & $0.987-1.022$ & 0.5683 \\
\hline ALT (per one IU /L) & 0.990 & $0.972-1.008$ & 0.3002 \\
\hline Child-Pugh score (per one point) & 1.270 & $1.020-1.520$ & 0.0424 \\
\hline Serum sodium (per one mmol/L) & 0.912 & $0.807-1.033$ & 0.1481 \\
\hline Serum creatinine (per one $\mathrm{mg} / \mathrm{dL}$ ) & 1.104 & $0.769-1.397$ & 0.4914 \\
\hline
\end{tabular}

\subsection{Univariate and Multivariate Analyses of Parameters Contributing to OS for Females}

Univariate analysis identified the following parameters as significantly associated with OS for females: age $(p=0.0214)$; SMI $(p=0.0076)$; Child-Pugh score $(p=0.0009)$; ALT $(p=0.0104)$; serum albumin ( $p=0.0003)$; prothrombin time (PT) $(p=0.0355)$; platelet count $(p=0.0333)$; triglyceride $(p=0.0011)$; serum creatinine $(p=0.0137) ; \operatorname{BMI}(p=0.0270)$ and presence of ascites $(p<0.0001)$ (Table 3a). As the Child-Pugh score includes serum albumin, PT, and ascites, they were not entered 
into the multivariate analysis, and because BMI significantly correlated with SMI, it was also excluded in the multivariate analysis to avoid the effect of collinearity. The HRs and $95 \%$ CIs calculated by using multivariate analysis for the seven significant variables $(p<0.05)$ in the univariate analysis are presented in Table $3 \mathrm{~b}$. SMI $(p=0.0016)$ and Child-Pugh scores $(p<0.0001)$ were found to be significant predictors related to OS in the multivariate analysis (Table $3 b$ ).

Table 3. (a) Univariate analyses of factors linked to overall survival for female $(n=178)$. (b) Multivariate analyses of factors linked to overall survival for female.

\begin{tabular}{|c|c|c|c|}
\hline \multicolumn{4}{|c|}{ (a) } \\
\hline \multirow{2}{*}{ Variables } & \multirow{2}{*}{\multicolumn{2}{|c|}{ Number of Each Category }} & Univariate \\
\hline & & & $p$ value \\
\hline Age (years) $\geq 77$, yes $/$ no & \multicolumn{2}{|c|}{$22 / 156$} & 0.0214 \\
\hline Cause of liver diseases, $\mathrm{B} / \mathrm{C} /$ others & \multicolumn{2}{|c|}{$10 / 113 / 55$} & 0.5037 \\
\hline $\mathrm{SMI} \geq 5.4 \mathrm{~cm}^{2} / \mathrm{m}^{2}$, yes $/$ no & \multicolumn{2}{|c|}{$139 / 39$} & 0.0076 \\
\hline Child-Pugh score $\geq 6$, yes $/$ no & \multicolumn{2}{|c|}{$98 / 80$} & 0.0009 \\
\hline $\mathrm{AST} \geq 80 \mathrm{IU} / \mathrm{L}$, yes $/$ no & \multicolumn{2}{|c|}{$20 / 158$} & 0.0544 \\
\hline $\mathrm{ALT} \geq 58 \mathrm{IU} / \mathrm{L}$, yes $/$ no & \multicolumn{2}{|c|}{$32 / 146$} & 0.0104 \\
\hline Serum albumin $\geq 3.4 \mathrm{~g} / \mathrm{dL}$, yes $/$ no & \multicolumn{2}{|c|}{$117 / 61$} & 0.0003 \\
\hline Total bilirubin $\geq 2.3 \mathrm{mg} / \mathrm{dL}$, yes $/ \mathrm{no}$ & \multicolumn{2}{|c|}{$10 / 168$} & 0.7607 \\
\hline Prothrombin time $\geq 73.7 \%$, yes $/$ no & \multicolumn{2}{|c|}{$103 / 75$} & 0.0355 \\
\hline Platelet count $\geq 9.7 \times 10^{4} / \mathrm{mm}^{3}$, yes $/$ no & \multicolumn{2}{|c|}{$92 / 86$} & 0.0333 \\
\hline Total cholesterol $\geq 176 \mathrm{mg} / \mathrm{dL}$, yes $/$ no & \multicolumn{2}{|c|}{$49 / 129$} & 0.0558 \\
\hline Triglyceride $\geq 72 \mathrm{mg} / \mathrm{dL}$, yes $/$ no & \multicolumn{2}{|c|}{$108 / 70$} & 0.0011 \\
\hline Serum sodium $\geq 139 \mathrm{mmol} / \mathrm{L}$, yes $/ \mathrm{no}$ & \multicolumn{2}{|c|}{$143 / 35$} & 0.4064 \\
\hline Fasting blood glucose $\geq 89 \mathrm{mg} / \mathrm{dL}$, yes $/$ no & \multicolumn{2}{|c|}{$142 / 36$} & 0.2428 \\
\hline Serum creatinine $\geq 0.63 \mathrm{mg} / \mathrm{dL}$, yes $/$ no & \multicolumn{2}{|c|}{$66 / 112$} & 0.0137 \\
\hline Body mass index $\geq 23.4 \mathrm{~kg} / \mathrm{m}^{2}$, yes $/$ no & \multicolumn{2}{|c|}{$78 / 100$} & 0.0270 \\
\hline Ascites, yes/no & \multicolumn{2}{|c|}{$19 / 159$} & $<0.0001$ \\
\hline \multicolumn{4}{|c|}{ (b) } \\
\hline \multirow{2}{*}{ Variables } & \multicolumn{3}{|c|}{ Multivariate Analysis } \\
\hline & Hazard ratio & $95 \% \mathrm{CI}$ & $p$ Value \\
\hline Age (per one year) & 1.018 & $0.977-1.063$ & 0.3998 \\
\hline SMI (per one $\mathrm{cm}^{2} / \mathrm{m}^{2}$ ) & 0.450 & $0.270-0.731$ & 0.0016 \\
\hline $\operatorname{ALT}($ per one IU/L) & 0.987 & $0.967-1.107$ & 0.0506 \\
\hline Platelet count (per one $\times 10^{4} / \mathrm{mm}^{3}$ ) & 0.919 & $0.828-1.008$ & 0.0943 \\
\hline Child-Pugh score (per one point) & 1.938 & $1.400-2.690$ & $<0.0001$ \\
\hline Triglyceride (per one mg/dL) & 0.998 & $0.983-1.011$ & 0.7760 \\
\hline Serum creatinine (per one $\mathrm{mg} / \mathrm{dL}$ ) & 2.546 & $0.413-10.860$ & 0.2545 \\
\hline
\end{tabular}

CI: confidence interval; SMI: skeletal muscle mass index; AST: aspartate aminotransferase; ALT: alanine aminotransferase.

\subsection{Time-Dependent ROC Analyses for OS in Males}

Results for time-dependent ROC analyses at two-, three-, four-, five-, six-, and seven-years of SMI and the Child-Pugh scores for males are shown in Figure 4A. All AUROCs for SMI at each time point were higher than those for Child-Pugh scores, denoting that SMI had consistently superior predictive ability for OS over Child-Pugh scores. 
(A)

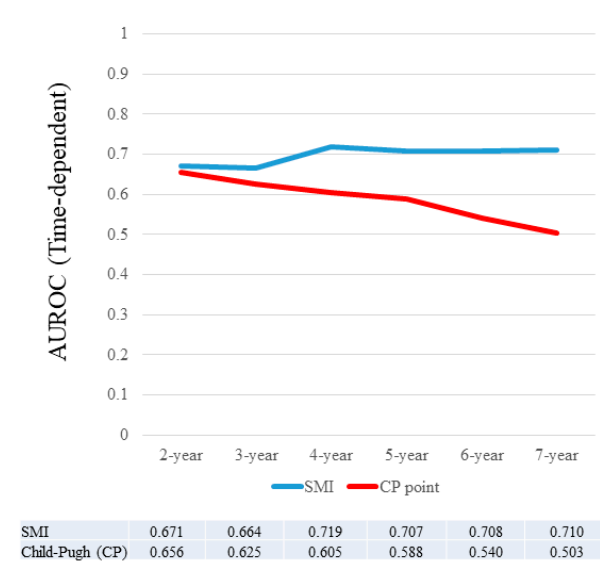

(B)

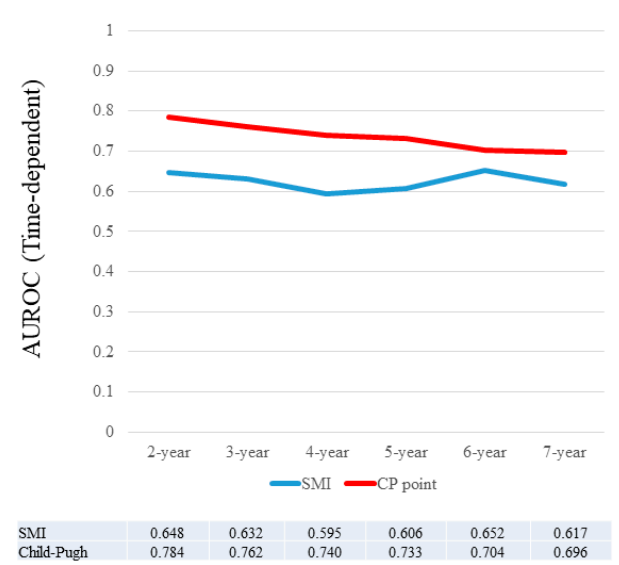

Figure 4. (A) Results for time-dependent ROC analyses at two-, three, four-, five-, six-, and seven-years of SMI and Child-Pugh scores for males; (B) Results for time-dependent ROC analyses at two-, three-, four-, five-, six-, and seven-years of SMI and Child-Pugh scores for females. ROC; receiver operating characteristics, AUROC; area under the ROC, SMI; skeletal muscle mass index, CP; Child-Pugh.

\subsection{Time-Dependent ROC Analyses for OS in Females}

Results for time-dependent ROC analyses at two-, three-, four-, five-, six-, and seven-years of SMI and Child-Pugh score for female were shown in Figure 4B. All AUROCs for Child-Pugh scores at each time point were higher than those for SMI, denoting that Child-Pugh scores had consistently superior predictive ability for OS over SMI.

\subsection{Time-Dependent ROC Analyses for OS in Male Patients with Child-Pugh A}

Results for time-dependent ROC analyses at two-, three-, four-, and five-years of SMI and Child-Pugh scores for male Child-Pugh A patients $(n=148)$ are shown in Figure 5A. All AUROCs for SMI in each time point were higher than those for Child-Pugh scores, denoting that SMI had consistently superior predictive ability for OS over Child-Pugh scores.

(A)

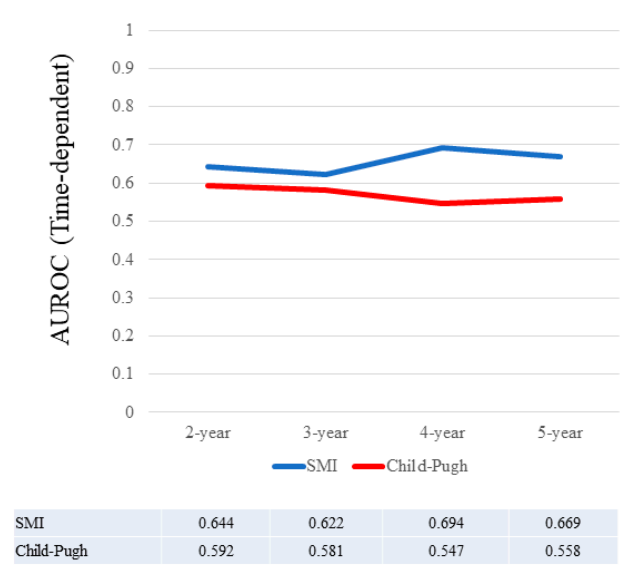

(B)

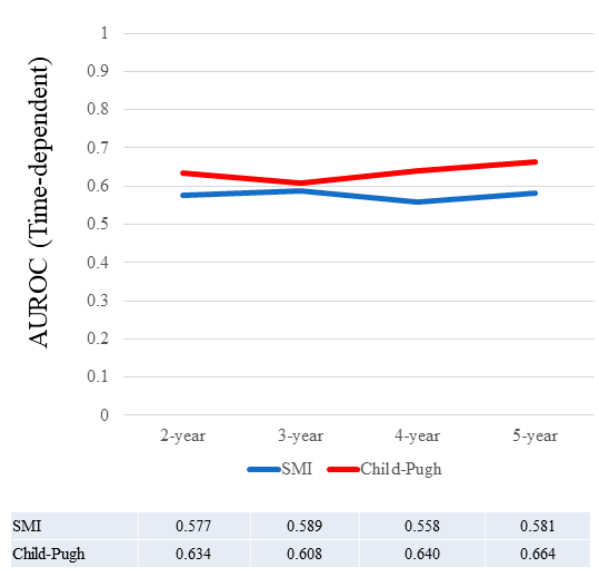

Figure 5. (A) Results for time-dependent ROC analyses at two-, three-, four-, and five-years of SMI and Child-Pugh scores for male Child-Pugh A patients; (B) Results for time-dependent ROC analyses at two-, three-, four-, and five-years of SMI and Child-Pugh scores for female Child-Pugh A patients. ROC; receiver operating characteristics, AUROC; area under the ROC, SMI; skeletal muscle mass index. 


\subsection{Time-Dependent ROC Analyses for OS in Female Patients with Child-Pugh A}

Results for time-dependent ROC analyses at two-, three-, four-, and five-years of SMI and Child-Pugh scores for female Child-Pugh A patients $(n=131)$ are shown in Figure 5B. All AUROCs for Child-Pugh score in each time point were higher than those for SMI, denoting that Child-Pugh scores had consistently superior predictive ability for OS over SMI.

\subsection{Time-Dependent ROC Analyses for OS in Male Patients with Child-Pugh B or C}

Results for time-dependent ROC analyses at two-, three-, four-, and five-years of SMI and Child-Pugh scores for male Child-Pugh B or C patients $(n=57)$ are shown in Figure 6A. All AUROCs for SMI at each time point were higher than those for Child-Pugh scores, denoting that SMI had consistently superior predictive ability for OS over Child-Pugh scores.
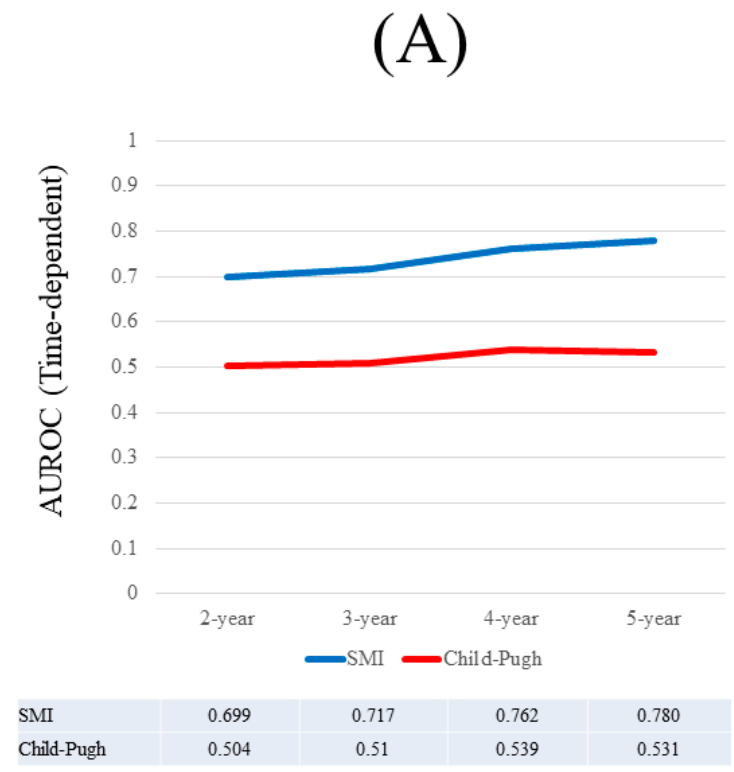

(B)

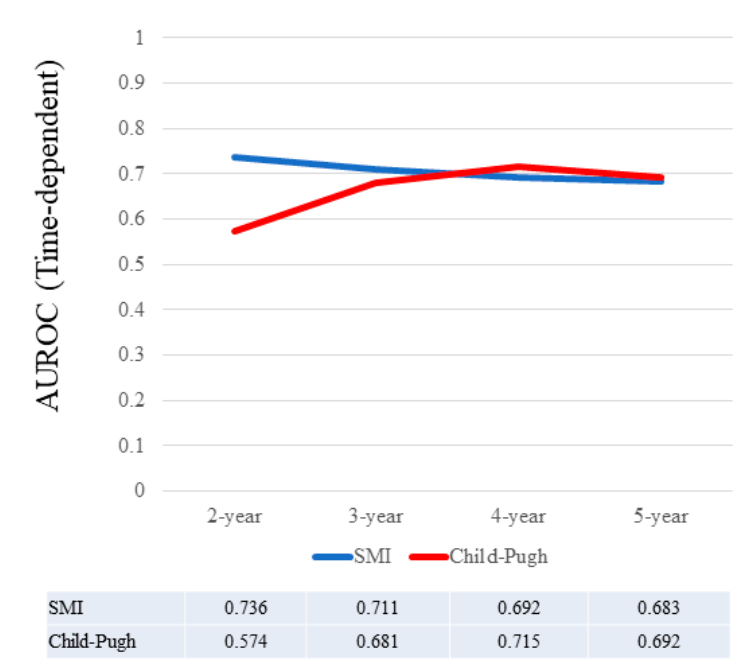

Figure 6. (A) Results for time-dependent ROC analyses at two-, three-, four-, and five-years of SMI and Child-Pugh score for male Child-Pugh B or C patients; (B) Results for time-dependent ROC analyses at two-, three-, four-, and five-years of SMI and Child-Pugh scores for female Child-Pugh B or C patients. ROC; receiver operating characteristics, AUROC; area under the ROC, SMI; skeletal muscle mass index.

\subsection{Time-Dependent ROC Analyses for OS in Female Patients with Child-Pugh B or C}

Results for time-dependent ROC analyses at two-, three-, four-, and five-years of SMI and Child-Pugh scores for female Child-Pugh B or C patients $(n=47)$ are shown in Figure 6B. At four- and five-years, Child-Pugh scores had higher AUROC than SMI.

\section{Discussion}

To the best of our knowledge, this is the first comparative study in SMI and Child-Pugh scores on clinical outcomes in LC patients. The Child-Pugh scoring system is a well-established prognostic system in LC patients [5-9]. While, SMI in LC has been currently attracting much attention owing to its well predictive performance $[14-17,19,27,28]$. Investigation into the influence of SMI on outcomes in LC patients is pivotal and, particularly, clarifying which of these two markers has stronger predictive impact for LC is clinically essential in light of creating a novel prognostic system in LC patients. We, therefore, conducted this comparative analysis to address this question. Since skeletal muscle mass significantly can differ between male and female, we analyzed and discussed separately in males and females. 
In our results, for males, SMI and Child-Pugh scores were revealed to be significant for OS in the multivariate analysis, and all AUROCs for SMI at each time point were higher than those for Child-Pugh scores. Additionally, in subgroup analyses for male patients with Child-Pugh A or Child-Pugh B or C, SMI had consistently higher AUROCs than the Child-Pugh score. For females, similar results were obtained in the multivariate analysis, however, all AUROCs for Child-Pugh scores at each time point were higher than those for SMI. These results denote that SMI as a predictor can perform well as compared with Child-Pugh score at least in male LC patients. Our current results may provide new information and shed some lights for the better comprehension of prognosis in LC patients. The significant difference in baseline characteristics in such as SMI, age, serum sodium, and serum creatinine between males and females can explain the different results according to gender. In particular, aging can be a significant predictor in LC patients $[14,39,40]$. Our country is an aging country $[39,40]$. The reasons for results in time-dependent ROC analysis in females may be attributed to our results that SMI did not significantly correlate with age in females.

In view of our current results, some interventional therapies, including BCAA therapy, testosterone therapy, or exercise, can be considered especially in male LC patients with lower skeletal muscle mass for ameliorating prognosis [13,35,41-43]. However, firm recommendations for LC patients are not currently available. As for cut-off value for BIA, AWGS recommends 2SDs below the average muscle mass of young adults $\left(7.0 \mathrm{~cm}^{2} / \mathrm{m}^{2}\right.$ for male and $5.7 \mathrm{~cm}^{2} / \mathrm{m}^{2}$ for female) [28]. In our outcome based ROC analysis, the optimal cut-off points for survival were $7.0 \mathrm{~cm}^{2} / \mathrm{m}^{2}$ for males and $5.4 \mathrm{~cm}^{2} / \mathrm{m}^{2}$ for females, which were quite similar to recommendations in AWGS and their recommendations were well validated in our analysis. From the viewpoint of outcome-based analysis, our results may be worthy of reporting.

As described above, it is of note that SMI significantly correlated with age in males, but not in females. In general, changes in muscle mass occur with aging and muscle mass loss is a common condition which is recognized as a part of aging [44]. However, our results showed that the rates of muscle mass decline can vary according to gender, suggesting that factors other than aging, such as diet intake and lifestyle, may influence the maintenance of healthy muscle mass [45]. On the other hand, although the liver transplantation allocation system utilizes MELD score to prioritize organs to the most ill subjects, MELD scores do not perform better than Child-Pugh scores in non-transplant settings. In other words, MELD scores can perform well in decompensated LC rather than compensated LC [8]. Due to the high proportion of patients with Child-Pugh A in our cohort, we did not include MELD scores in the analysis.

Several limitations must be acknowledged in our current analysis. First, this study is a single-center retrospective observational study utilizing data for BIA and parameters reflecting muscle function such as hang grip strength or walking speed were not assessed in this analysis. In future studies, both skeletal muscle mass and muscle function should be evaluated in outcome based analyses. Second, patients with severe ascites were excluded from our analysis because SMI can be overestimated in these patients. Thus, the number of Child-Pugh $C$ patients was rather small and our results cannot be adapted to such patients. Body composition analyses using BIA can be challenging in LC patients with severe ascites. Finally, various treatments for underlying liver diseases were performed during follow-up period in each patient, potentially creating bias. However, our study results denoted that SMI had higher predictive ability, at least in male LC patients. Results in time-dependent ROC analysis support our assertion for the predictive superiority of SMI over Child-Pugh scores in males.

In conclusion, SMI can be accessible for predicting outcomes, at least in male LC patients. Some interventions for male patients with lower SMI may be recommended.

Acknowledgments: The authors would like to thank all medical staff in our hospital for data collection. Financial support is none.

Author Contributions: Hiroki Nishikawa and Hirayuki Enomoto analyzed the data and wrote the paper. Shuhei Nishiguchi supervised this work. The other remaining authors collected data.

Conflicts of Interest: The authors declare no conflict of interest. 


\section{Abbreviations}

LC: liver cirrhosis; MELD: Model for End-stage Liver Disease; LT: liver transplantation; HCC: hepatocellular carcinoma; CT: computed tomography; MRI: magnetic resonance imaging; BIA: bioimpedance analysis; SMI: skeletal muscle mass index; BMI: body mass index; US: ultrasonography; BCAA: branched-chain amino acid; ROC: receiver operating characteristic curve; OS: overall survival; AUROC: area under the receiver operating characteristic curve; SD: standard deviation; AWGS: Asian Working Group for Sarcopenia criteria; D-SMI: decreased SMI; ALT: alanine aminotransferase; HR: hazard ratios; CI: confidence interval.

\section{References}

1. Cheung, K.; Lee, S.S.; Raman, M. Prevalence and mechanisms of malnutrition in patients with advanced liver disease, and nutrition management strategies. Clin. Gastroenterol. Hepatol. 2012, 10, 117-125. [CrossRef] [PubMed]

2. Charlton, M.R. Branched-chain amino acid enriched supplements as therapy for liver disease. J. Nutr. 2006, 136 (Suppl. 1), 295S-298S. [CrossRef]

3. Kawaguchi, T.; Izumi, N.; Charlton, M.R.; Sata, M. Branched-chain amino acids as pharmacological nutrients in chronic liver disease. Hepatology 2011, 54, 1063-1070. [CrossRef] [PubMed]

4. Fukui, H.; Saito, H.; Ueno, Y.; Uto, H.; Obara, K.; Sakaida, I.; Shibuya, A.; Seike, M.; Nagoshi, S.; Segawa, M.; et al. Evidence-based clinical practice guidelines for liver cirrhosis 2015. J. Gastroenterol. 2016, 51, 629-650. [CrossRef] [PubMed]

5. Kamath, P.S.; Wiesner, R.H.; Malinchoc, M.; Kremers, W.; Therneau, T.M.; Kosberg, C.L.; D'Amico, G.; Dickson, E.R.; Kim, W.R. A model to predict survival in patients with end-stage liver disease. Hepatology 2001, 33, 464-470. [CrossRef] [PubMed]

6. Kim, W.R.; Biggins, S.W.; Kremers, W.K.; Wiesner, R.H.; Kamath, P.S.; Benson, J.T.; Edwards, E.; Therneau, T.M. Hyponatremia and mortality among patients on the liver-transplant waiting list. N. Engl. J. Med. 2008, 359, 1018-1026. [CrossRef] [PubMed]

7. D'Amico, G.; Garcia-Tsao, G.; Pagliaro, L. Natural history and prognostic indicators of survival in cirrhosis: A systematic review of 118 studies. J. Hepatol. 2006, 44, 217-231. [CrossRef] [PubMed]

8. Cholongitas, E.; Papatheodoridis, G.V.; Vangeli, M.; Terreni, N.; Patch, D.; Burroughs, A.K. Systematic review: The model for end-stage liver disease-Should it replace Child-Pugh's classification for assessing prognosis in cirrhosis? Aliment. Pharmacol. Ther. 2005, 22, 1079-1089. [CrossRef] [PubMed]

9. Botta, F.; Giannini, E.; Romagnoli, P.; Fasoli, A.; Malfatti, F.; Chiarbonello, B.; Testa, E.; Risso, D.; Colla, G.; Testa, R. MELD scoring system is useful for predicting prognosis in patients with liver cirrhosis and is correlated with residual liver function: A European study. Gut 2003, 52, 134-139. [CrossRef] [PubMed]

10. Periyalwar, P.; Dasarathy, S. Malnutrition in cirrhosis: Contribution and consequences of sarcopenia on metabolic and clinical responses. Clin. Liver Dis. 2012, 16, 95-131. [CrossRef] [PubMed]

11. Cruz-Jentoft, A.J.; Landi, F.; Schneider, S.M.; Zúñiga, C.; Arai, H.; Boirie, Y.; Chen, L.K.; Fielding, R.A.; Martin, F.C.; Michel, J.P.; et al. Prevalence of and interventions for sarcopenia in ageing adults: A systematic review. Report of the International Sarcopenia Initiative (EWGSOP and IWGS). Age Ageing 2014, 43, 748-759. [CrossRef] [PubMed]

12. Sinclair, M.; Gow, P.J.; Grossmann, M.; Angus, P.W. Review article: Sarcopenia in cirrhosis-Aetiology, implications and potential therapeutic interventions. Aliment. Pharmacol. Ther. 2016, 43, 765-777. [CrossRef] [PubMed]

13. Hanai, T.; Shiraki, M.; Nishimura, K.; Ohnishi, S.; Imai, K.; Suetsugu, A.; Takai, K.; Shimizu, M.; Moriwaki, H. Sarcopenia impairs prognosis of patients with liver cirrhosis. Nutrition 2015, 31, 193-199. [CrossRef] [PubMed]

14. Nishikawa, H.; Shiraki, M.; Hiramatsu, A.; Moriya, K.; Hino, K.; Nishiguchi, S. JSH guidelines for sarcopenia in liver disease (first edition): Recommendation from the working group for creation of sarcopenia assessment criteria in the JSH. Hepatol. Res. 2016, 46, 951-963. [CrossRef] [PubMed]

15. Santilli, V.; Bernetti, A.; Mangone, M.; Paoloni, M. Clinical definition of sarcopenia. Clin. Cases Miner. Bone Metab. 2014, 11, 177-180. [CrossRef] [PubMed]

16. Nardelli, S.; Lattanzi, B.; Torrisi, S.; Greco, F.; Farcomeni, A.; Gioia, S.; Merli, M.; Riggio, O. Sarcopenia is Risk Factor for Development of Hepatic Encephalopathy After Transjugular Intrahepatic Portosysthemic Shunt Placement. Clin. Gastroenterol. Hepatol. 2017, 15, 934-936. [CrossRef] [PubMed] 
17. Itoh, S.; Shirabe, K.; Matsumoto, Y.; Yoshiya, S.; Muto, J.; Harimoto, N.; Yamashita, Y.; Ikegami, T.; Yoshizumi, T.; Nishie, A.; et al. Effect of body composition on outcomes after hepatic resection for hepatocellular carcinoma. Ann. Surg. Oncol. 2014, 21, 3063-3068. [CrossRef] [PubMed]

18. Carrara, G.; Pecorelli, N.; De Cobelli, F.; Cristel, G.; Damascelli, A.; Beretta, L.; Braga, M. Preoperative sarcopenia determinants in pancreatic cancer patients. Clin. Nutr. 2016. [CrossRef] [PubMed]

19. Fujiwara, N.; Nakagawa, H.; Kudo, Y.; Tateishi, R.; Taguri, M.; Watadani, T.; Nakagomi, R.; Kondo, M.; Nakatsuka, T.; Minami, T.; et al. Sarcopenia, intramuscular fat deposition, and visceral adiposity independently predict the outcomes of hepatocellular carcinoma. J. Hepatol. 2015, 63, 131-140. [CrossRef] [PubMed]

20. Zhuang, C.L.; Huang, D.D.; Pang, W.Y.; Zhou, C.J.; Wang, S.L.; Lou, N.; Ma, L.L.; Yu, Z.; Shen, X. Sarcopenia is an Independent Predictor of Severe Postoperative Complications and Long-Term Survival after Radical Gastrectomy for Gastric Cancer: Analysis from a Large-Scale Cohort. Medicine 2016, 95, e3164. [CrossRef] [PubMed]

21. Harimoto, N.; Shirabe, K.; Yamashita, Y.I.; Ikegami, T.; Yoshizumi, T.; Soejima, Y.; Ikeda, T.; Maehara, Y.; Nishie, A.; Yamanaka, T. Sarcopenia as a predictor of prognosis in patients following hepatectomy for hepatocellular carcinoma. Br. J. Surg. 2013, 100, 1523-1530. [CrossRef] [PubMed]

22. Antoun, S.; Borget, I.; Lanoy, E. Impact of sarcopenia on the prognosis and treatment toxicities in patients diagnosed with cancer. Curr. Opin. Support Palliat. Care 2013, 7, 383-389. [CrossRef] [PubMed]

23. Iritani, S.; Imai, K.; Takai, K.; Hanai, T.; Ideta, T.; Miyazaki, T.; Suetsugu, A.; Shiraki, M.; Shimizu, M.; Moriwaki, H. Skeletal muscle depletion is an independent prognostic factor for hepatocellular carcinoma. J. Gastroenterol. 2015, 50, 323-332. [CrossRef] [PubMed]

24. Kamachi, S.; Mizuta, T.; Otsuka, T.; Nakashita, S.; Ide, Y.; Miyoshi, A.; Kitahara, K.; Eguchi, Y.; Ozaki, I.; Anzai, K. Sarcopenia is a risk factor for the recurrence of hepatocellular carcinoma after curative treatment. Hepatol. Res. 2016, 46, 201-208. [CrossRef] [PubMed]

25. Suzuki, Y.; Okamoto, T.; Fujishita, T.; Katsura, M.; Akamine, T.; Takamori, S.; Morodomi, Y.; Tagawa, T.; Shoji, F.; Maehara, Y. Clinical implications of sarcopenia in patients undergoing complete resection for early non-small cell lung cancer. Lung Cancer 2016, 101, 92-97. [CrossRef] [PubMed]

26. Harimoto, N.; Yoshizumi, T.; Shimokawa, M.; Sakata, K.; Kimura, K.; Itoh, S.; Ikegami, T.; Ikeda, T.; Shirabe, K.; Maehara, Y. Sarcopenia is a poor prognostic factor following hepatic resection in patients 70 years of age and older with hepatocellular carcinoma. Hepatol. Res. 2016, 46, 1247-1255. [CrossRef] [PubMed]

27. Hamaguchi, Y.; Kaido, T.; Okumura, S.; Kobayashi, A.; Hammad, A.; Tamai, Y.; Inagaki, N.; Uemoto, S. Proposal for new diagnostic criteria for low skeletal muscle mass based on computed tomography imaging in Asian adults. Nutrition 2016, 32, 1200-1205. [CrossRef] [PubMed]

28. Chen, L.K.; Liu, L.K.; Woo, J.; Assantachai, P.; Auyeung, T.W.; Bahyah, K.S.; Chou, M.Y.; Chen, L.Y.; Hsu, P.S.; Krairit, O.; et al. Sarcopenia in Asia: Consensus Report of the Asian Working Group for Sarcopenia. J. Am. Med. Dir. Assoc. 2014, 15, 95-101. [CrossRef] [PubMed]

29. Dasarathy, S. Consilience in sarcopenia of cirrhosis. J. Cachexia Sarcopenia Muscle 2012, 3, 225-237. [CrossRef] [PubMed]

30. Montano-Loza, A.J. Clinical relevance of sarcopenia in patients with cirrhosis. World J. Gastroenterol. 2014, 20, 8061-8071. [CrossRef] [PubMed]

31. Kudo, M.; Zheng, R.Q.; Kim, S.R.; Okabe, Y.; Osaki, Y.; Iijima, H.; Itani, T.; Kasugai, H.; Kanematsu, M.; Ito, K.; et al. Diagnostic accuracy of imaging for liver cirrhosis compared to histologically proven liver cirrhosis. A multicenter collaborative study. Intervirology 2008, 51, 17-26. [CrossRef] [PubMed]

32. Zarski, J.P.; Sturm, N.; Guechot, J.; Paris, A.; Zafrani, E.S.; Asselah, T.; Boisson, R.C.; Bosson, J.L.; Guyader, D.; Renversez, J.C.; et al. Comparison of nine blood tests and transient elastography for liver fibrosis in chronic hepatitis C: The ANRS HCEP-23 study. J. Hepatol. 2012, 56, 55-62. [CrossRef] [PubMed]

33. Tsochatzis, E.A.; Gurusamy, K.S.; Ntaoula, S.; Cholongitas, E.; Davidson, B.R.; Burroughs, A.K. Elastography for the diagnosis of severity of fibrosis in chronic liver disease: A meta-analysis of diagnostic accuracy. J. Hepatol. 2011, 54, 650-659. [CrossRef] [PubMed]

34. Kumada, H.; Okanoue, T.; Onji, M.; Moriwaki, H.; Izumi, N.; Tanaka, E.; Chayama, K.; Sakisaka, S.; Takehara, T.; Oketani, M.; et al. Guidelines for the treatment of chronic hepatitis and cirrhosis due to hepatitis C virus infection for the fiscal year 2008 in Japan. Hepatol. Res. 2010, 40, 8-13. [CrossRef] [PubMed] 
35. Kappus, M.R.; Mendoza, M.S.; Nguyen, D.; Medici, V.; McClave, S.A. Sarcopenia in Patients with Chronic Liver Disease: Can It Be Altered by Diet and Exercise? Curr. Gastroenterol. Rep. 2016, 18, 43. [CrossRef] [PubMed]

36. Heagerty, P.J.; Zheng, Y. Survival model predictive accuracy and ROC curves. Biometrics 2005, 61, 92-105. [CrossRef] [PubMed]

37. European Association for the Study of the Liver; European Organisation for Research and Treatment of Cancer. EASL-EORTC Clinical Practice guidelines: Management of hepatocellular carcinoma. J. Hepatol. 2012, 56, 908-943.

38. Kudo, M.; Izumi, N.; Kokudo, N.; Matsui, O.; Sakamoto, M.; Nakashima, O.; Kojiro, M.; Makuuchi, M.; HCC Expert Panel of Japan Society of Hepatology. Management of hepatocellular carcinoma in Japan: Consensus-Based Clinical Practice Guidelines proposed by the Japan Society of Hepatology (JSH) 2010 updated version. Dig Dis. 2011, 29, 339-364. [CrossRef] [PubMed]

39. Asahina, Y.; Tsuchiya, K.; Tamaki, N.; Hirayama, I.; Tanaka, T.; Sato, M.; Yasui, Y.; Hosokawa, T.; Ueda, K.; Kuzuya, T.; et al. Effect of aging on risk for hepatocellular carcinoma in chronic hepatitis $\mathrm{C}$ virus infection. Hepatology 2010, 52, 518-527. [CrossRef] [PubMed]

40. Osaki, Y.; Nishikawa, H. Treatment for hepatocellular carcinoma in Japan over the last three decades: Our experience and published work review. Hepatol Res. 2015, 45, 59-74. [CrossRef] [PubMed]

41. Davuluri, G.; Krokowski, D.; Guan, B.J.; Kumar, A.; Thapaliya, S.; Singh, D.; Hatzoglou, M.; Dasarathy, S. Metabolic adaptation of skeletal muscle to hyperammonemia drives the beneficial effects of l-leucine in cirrhosis. J. Hepatol. 2016, 65, 929-937. [CrossRef] [PubMed]

42. Sinclair, M.; Grossmann, M.; Hoermann, R.; Angus, P.W.; Gow, P.J. Testosterone therapy increases muscle mass in men with cirrhosis and low testosterone: A randomised controlled trial. J. Hepatol. 2016, 65, 906-913. [CrossRef] [PubMed]

43. Dasarathy, S. Cause and management of muscle wasting in chronic liver disease. Curr. Opin. Gastroenterol. 2016, 32, 159-165. [CrossRef] [PubMed]

44. St-Onge, M.P. Relationship between body composition changes and changes in physical function and metabolic risk factors in aging. Curr. Opin. Clin. Nutr. Metab. Care 2005, 8, 523-528. [PubMed]

45. Syddall, H.; Evandrou, M.; Cooper, C.; Sayer, A.A. Social inequalities in grip strength, physical function, and falls among community dwelling older men and women: Findings from the Hertfordshire Cohort Study. J. Aging Health 2009, 21, 913-939. [CrossRef] [PubMed] 